\title{
Mechanical Properties of Poly(ethylene terephthalate) Estimated in Terms of Orientation Distribution of Crystallites and Amorphous Chain Segments under Simultaneous Biaxially Stretching
}

\author{
Yuezhen BIN, $^{\dagger}$ Kumiko OISHI, Kyoko YoshidA, and Masaru MATsUO ${ }^{\dagger}$ \\ Textile and Apparel Science, Faculty of Human Life and Environment, Nara Women's University, \\ Kitauoya Nishi-machi, Nara 630-8263, Japan
}

(Received May 11, 2004; Accepted August 27, 2004; Published November 15, 2004)

\begin{abstract}
Young's modulus of poly(ethylene terephthalate) (PET) film was estimated by using the generalized orientation factors of crystallites and amorphous chain segments calculated from the orientation functions of crystallites and amorphous chain segments. Theoretical analysis was carried out on the basis of a two-phase model assuring the homogeneous stress hypothesis for a polycrystalline material. In this model system, the anisotropic crystal phase is surrounded by the anisotropic amorphous phase. As the theoretical values of elastic compliance of crystal unit of PET, the values by Tashiro were adopted. The experimental values of Young's modulus at room temperature were in good agreement with the predicted value calculated by using the theoretical compliance. Furthermore, the ultimate value of Young's modulus was estimated by assuming an ideal simultaneous biaxially stretching film with $100 \%$ crystallinity and the perfect orientation of the $c$-axis as well as that of benzene rings parallel to the film surface. The predicted ultimate goal of the Young's modulus was less than $5 \mathrm{GPa}$ indicating the difficulty in producing high modulus and high strength PET sheets in terms of theoretical aspects. [DOI 10.1295/polymj.36.888]

KEY WORDS Young's Modulus / Poly(ethylene terephthalate) / Generalized Orientation Factors / Simultaneous Biaxially Stretching Film / Two-phase Model /
\end{abstract}

Young's modulus of a polycrystalline material can be generally estimated by using the generalized fourth and second order orientation factors by using the method of Roe and Krigbaum ${ }^{1,2}$ and crystallinity on the basis of a two-phase model, ${ }^{7,8}$ assuming the homogeneous stress within the material. ${ }^{8,9}$ This treatment represented as a continuous body is essentially suitable, if there exist no aggregations of crystallites such as spherulites and rods within the material. The analysis has been successful for polyethylene (PE) with an orthorhombic crystal unit ${ }^{5,6}$ and for poly(vinyl alcohol) (PVA), ${ }^{10}$ nylon $6^{11,12}$ and cellulose ${ }^{13}$ with a monoclinic crystal unit. The generalized fourth order orientation factors can be obtained from the orientation factors. In doing so, at least, the orientation functions of the reciprocal lattice vectors of five crystal planes are needed for PVA and that of three crystal planes are needed for PE. However, there is no report for the estimation of poly(ethylene terephthalate) (PET) and poly(buthylene terephthalate) (PBT), because of difficulty in estimating the orientation functions of nine crystal planes of PET and PBT with a triclinic crystal unit.

In previous papers, ${ }^{14,15}$ the orientation of crystallites as well as the orientation of the three principal crystallographic axes, the $a-, b$-, and $c$-axes, were estimated in terms of the orientation distribution functions for a PBT film ${ }^{14}$ stretched uniaxially and for a PET film stretched biaxially. ${ }^{15}$ The successful results predicted that the preferential orientation function of the $c$-axis with respect to the stretching direction follows a floating model associated with an affine fashion. ${ }^{16}$ Furthermore, the same method was applied to a simultaneous biaxially stretched PET film. ${ }^{15}$ As for the PET film, the orientation distribution function provided the information that most of benzene rings in a main chain of PET are oriented parallel to the film surface but some of them are oriented perpendicular to the film surface.

This paper is concerned with the rigorous estimation of Young's modulus of PET by using the orientation distribution function obtained in the previous experiment. Great contribution to the treatment was done by Hibi et al. ${ }^{17,18}$ They tried to calculate Young's modulus in relation to the generalized orientation factors and crystallinity. Based on their concept, the actual calculation is carried out using the generalized orientation factors with the fourth and second orders estimated from the orientation distribution function of crystallites in the previous paper, ${ }^{15}$ on the basis of a twophase model ${ }^{3-7}$ assuring the homogeneous stress hypothesis for a polycrystalline material. ${ }^{8,9}$

\section{EXPERIMENTAL}

Original samples used in this experiment were amorphous PET film whose film thickness is $c a$.

${ }^{\dagger}$ To whom correspondence should be addressed (Tel \& Fax: 81-742-20-3462, E-mail: yuezhen@cc.nara-wu.ac.jp, m-matsuo@cc.nara-wu.ac.jp). 
Table I. Characteristics of PET specimens elngated at $95^{\circ} \mathrm{C}$ and annealed at $160^{\circ} \mathrm{C}$ for $30 \mathrm{~min}$

\begin{tabular}{ccc}
\hline Draw ratio & $\begin{array}{c}\text { Volume crystallinity } \\
(\%)\end{array}$ & $\begin{array}{c}\text { Melting point } \\
\left({ }^{\circ} \mathrm{C}\right)\end{array}$ \\
\hline 1 & 39.4 & 258 \\
$2 \times 2$ & 41.9 & 258 \\
$3 \times 3$ & 45.0 & 258 \\
$4 \times 4$ & 47.4 & 259 \\
\hline
\end{tabular}

$220 \mu \mathrm{m}$ and volume crystallinity is $0.3 \%$, which was used in the previous paper. ${ }^{15}$ The simultaneous biaxially elongation of the amorphous film was done up to the desired draw ratios. For the theoretical estimation, the results for the film with $4 \times 4$ folds reported in the previous paper ${ }^{15}$ were adopted in this paper. As described there the elongation was done at $95^{\circ} \mathrm{C}$ and then annealed at $160^{\circ} \mathrm{C}$ for $30 \mathrm{~min}$. The elongated sample was fixed under cooling process to $20^{\circ} \mathrm{C}$ and also maintained for $30 \mathrm{~min}$ at $25^{\circ} \mathrm{C}$. The characteristics of the PET film are summarized again in Table I.

Birefringence was measured between crossed polar in an optical microscope (Olympus 206648).

To check an outline for the mechanical properties of the simultaneous biaxially stretching PET film, the complex dynamic tensile modulus was measured at $10 \mathrm{~Hz}$ over the temperature range from -150 to 300 ${ }^{\circ} \mathrm{C}$ by using a visco-elastic spectrometer (VES-F) obtained from Iwamoto Machine Co., Ltd. The length of the specimen between the jaws was $40 \mathrm{~mm}$ and the width was about $1.5 \mathrm{~mm}$. During measurements, the specimen was subjected to a static tensile strain in order to place the sample in tension during the axial sinusoidal oscillation. The complex dynamic modulus was measured by imposing a small dynamic strain to ensure linear visco-elastic behavior of the specimen.

Figure 1 shows storage modulus $\left(E^{\prime}\right)$ and loss modulus $\left(E^{\prime \prime}\right)$ of the films elongated up to the desired draw ratio $(\lambda)$. The elongation condition is the same as described in the previous paper. ${ }^{15}$ The storage modulus increases with $\lambda$ indicating increases in molecular orientation and crystallinity. However, the increase in $E^{\prime}$ with increasing $\lambda$ is less significant in comparison with the increase under uniaxial stretching. ${ }^{19}$ This reason shall be discussed later. The loss modulus at each draw ratio shows two peaks. The results are similar to the results by Illers et al. ${ }^{20}$ which were reported for the temperature dependence of shear modulus $G^{\prime}$ and loss modulus $G^{\prime \prime}$ with a torsion pendulum at a constant frequency of 1 cycle/s in the temperature range between -180 and $260^{\circ} \mathrm{C}$. The peaks at higher and lower temperature sides are termed as $\beta$ and $\gamma$ relaxations. The NMR measurements by Ward et al. ${ }^{21}$ revealed clearly that above the $\beta$-dispersion temperature, both the benzene ring and the methylene group protons are under-
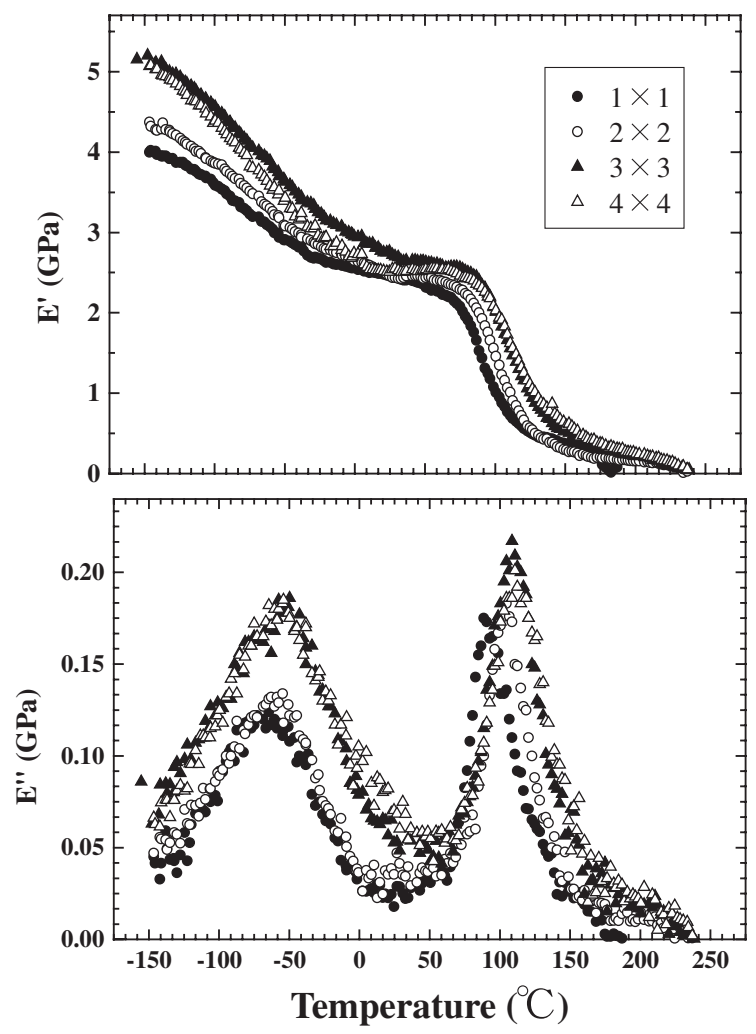

Figure 1. Temperature dependence of the storage and loss moduli of the PET films elongated biaxially up to the desired temperature.

going considerable molecular motion. Since then, the $\beta$-dispersion at $74^{\circ} \mathrm{C}$ has been explained as the contribution from the glass transition (the $\beta$-dispersion) associated with an increase in free volume and the micro-Brownian motion of the chain segments starting at this temperature. Incidentally, the existence of the $\alpha$-dispersion associated with crystal relaxation was found as a small peak by Yoshihara et al. ${ }^{22}$ The peak appeared at temperature close to the $\beta$-dispersion. The $\gamma$ peak of $E^{\prime \prime}$ is certainly built up from two or more closely adjacent peaks, belonging to motions of different groups or groups with different steric or energetic interaction. According to the NMR experiments by Ward et al. ${ }^{21}$ there are hindered rotations of methylene groups below glass transition temperature which presumably also occur within the crystalline regions.

To make clear the difference of mechanical properties between uniaxial and biaxial stretching films, the crystal lattice modulus were measured as a function of temperature for understanding intrinsic mechanical properties of PET crystallites. The X-ray diffraction intensity was measured with a sintillaion counter and a $12 \mathrm{~kW}$ rotating-anode $\mathrm{X}$-ray generator (Rigaku RDA-rA operated at $200 \mathrm{~mA}$ and $40 \mathrm{kV}$ ). The X-ray beam was monochromatized with a curved graphite monochromatar. Crystal strain at elevated temperature was provided by a constant-stretching apparatus 
which has been described elsewhere. ${ }^{19}$ For the measurements, the specimen was mounted vertically in the stretching clamps of the apparatus. The intensity distribution was measured with a step-scanning device at a step interval of $0.1^{\circ}$, each at a fixed time of $40 \mathrm{~s}$.

The measurements were carried out for the crystal lattice modulus along the reciprocal lattice vectors of the (105), (100), (010) and (110) planes. In doing so, uniaxial stretching specimens were used. The original specimen was first drawn up to 12 times uniaxially in a hot water bath at $95^{\circ} \mathrm{C}$ and after vacuum-drying the specimen was drawn to 20 times again in a hot oven at $250{ }^{\circ} \mathrm{C}$ under nitrogen in the second stage. The crystallinity was $c a$. $60 \%$. The crystal lattice modulus of PET along the chain direction cannot be observed directly by the X-ray diffraction, since there exists no detectable crystal plane whose reciprocal lattice vector is parallel to the crystal chain axis. In the unit cell of PET, the reciprocal lattice vector of the (105) plane, among all the crystal planes, most closely parallels the $c$-axis.

Table II shows the modulus of the crystal chain direction ( $c$-axis) estimated from the $U_{(\overline{1} 05)}$ as well as the crystal lattice moduli of the $U_{(010)}, U_{(\overline{1} 10)}$ and $U_{(100)}$ as a function of temperature. In addition, the Young's moduli in a bulk along the stretching direction and along the direction perpendicular to the stretching direction are listed as a function of temperature. Such experiments were carried out only to understand the different temperature dependence of crystal lattice modulus of PET. As listed in Table II, the crystal

Table II. Temperature dependence of the crystal moduli along the reciprocal lattice vectors and Young's moduli parallel $\left(E_{33}\right)$ and perpendicular $\left(E_{11}\right)$ to the uniaxial stretching direction (unit, GPa)

\begin{tabular}{rcccccc}
\hline $\begin{array}{r}\text { Temp } \\
\left({ }^{\circ} \mathrm{C}\right)\end{array}$ & $U_{(\overline{1} 05)}$ & $E_{33}$ & $U_{(010)}$ & $U_{(110)}$ & $U_{(100)}$ & $E_{11}$ \\
\hline 20 & 125 & 7.7 & 8.0 & 12.9 & 13.0 & 2.1 \\
50 & 123 & 7.6 & 7.6 & 12.6 & 12.9 & 2.0 \\
70 & 123 & 7.6 & 7.7 & 11.5 & 11.4 & 2.0 \\
80 & & & 6.7 & 8.9 & 9.1 & 1.6 \\
100 & 122 & 4.3 & 5.3 & 7.5 & 8.4 & 1.0 \\
120 & & & 5.4 & 7.2 & 9.1 & 0.7 \\
125 & 124 & 3.4 & & & & \\
140 & & & 5.1 & 5.8 & 8.7 & 0.6 \\
150 & 120 & 2.7 & & & & \\
160 & & & 5.1 & 5.5 & 7.9 & 0.5 \\
175 & 123 & 2.2 & & & & \\
180 & & & 4.9 & 5.0 & 7.9 & 0.4 \\
200 & 124 & & 4.6 & 4.5 & 7.4 & 0.3 \\
210 & 125 & & & & & \\
220 & 121 & & & & & \\
230 & 123 & & & & & \\
240 & 117 & & & & & \\
\hline
\end{tabular}

lattice modulus for $U_{(\overline{1} 05)}$ is almost independent of temperature. The values of the crystal lattice modulus of the (105) plane are in the range from 121 to $125 \mathrm{GPa}$ at temperatures lower than $230^{\circ} \mathrm{C}$. Therefore the crystal lattice modulus along the chain direction is $c a .118 \mathrm{GPa}$ on the basis of the theoretical analysis described elsewhere. ${ }^{19}$ This indicates no temperature dependence of crystal lattice modulus of PET along the chain direction, as has been reported for $\mathrm{PE}^{23}$ PP ${ }^{24}$ and PVA. ${ }^{10}$ In contrast, the other crystal moduli decrease with increasing temperature. Of course, temperature dependence of the crystal lattice moduli as listed in Table II is useful as fundamental information to analyze the temperature dependence of the present simultaneous biaxially stretching films later.

\section{RESULTS AND DISCUSSION}

In a paracrystalline material, a number of small crystallites as islands are buried in an anisotropic amorphous phase as ocean in the case that there are no large crystal aggregations such as spherulites and rods. Namely, a number of crystallites with each different orientation are surrounded by an anisotropic amorphous phase whose molecular chains are oriented in the stretching direction as shown in Figure 2a. Model (b) simplifies the morphology of (a) and represents each crystallite as an island showing a rectangular, in which the $X_{1}, X_{2}$, and $X_{3}$ axes are fixed in the bulk specimen. An arbitrary crystallite is generally represented as $\mu_{i} \zeta_{j} \delta_{k}$ and the total crystallites, as $\sum_{i} \sum_{j} \sum_{k} \mu_{i} \zeta_{j} \delta_{k}$, corresponding to crystallinity.

To pursue the theoretical calculation for estimating Young's modulus, further simplification of the model system in Figure $2 b$ is proposed as a composite model in Figure 3. In Figure 3, a crystal phase is represented as aggregation of a number of crystallites with different orientation distribution. Namely, the crystallites with different orientation is replaced as orientation
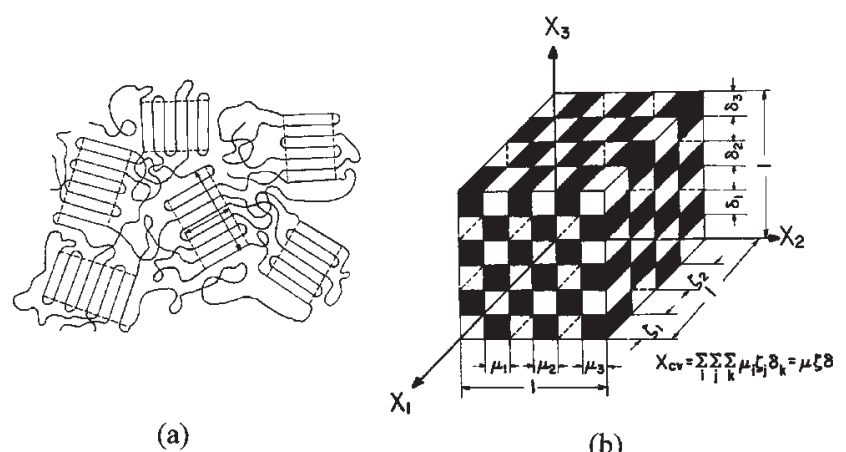

(b)

Figure 2. (a) A schematic model representing a number of crystallites with each different orientation surrounded by oriented amorphous phase. (b) A simplified model of (a), in which each crystallite with different orientation is represented by a rectangle. 


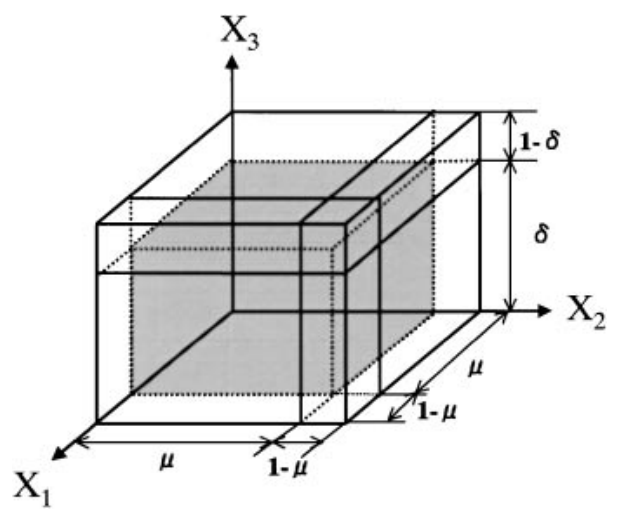

Figure 3. A composite model in which oriented crystallites are surrounded by an anisotropic amorphous phase.

distribution of crystallites in the crystal phase in Figure 3. In the proposed model, the anisotropic amorphous phase is adjacent to the oriented crystal phase with the interfaces perpendicular to the $X_{1}, X_{2}$ and $X_{3}$ axes. In the simultaneous biaxially stretching film, the $X_{1}$ and $X_{2}$ axes are chosen in the stretching direction and the $X_{3}$ axis, in the film thickness direction. This model system satisfies the morphological characteristics of a polymeric system with a low degree of molecular orientation and low crystallinity such as a simultaneous biaxial stretching film. Volume crystallinity $X_{\mathrm{cv}}$ is represented by $\delta \mu^{2}$ by the use of the fraction lengths $\delta$ and $\mu$ in the directions of the $X_{3}$ and $X_{2}$ (and $X_{1}$ ) axes because the present specimen is isotropic around the $X_{3}$ axis and then the $\mu=\zeta$. In this model system, amorphous layers are adjacent to the oriented crystalline layers with the interfaces perpendicular to the $X_{1}, X_{2}$ and $X_{3}$ axes. Strains of the two phases at the boundary are assumed to be identical. This model can be constructed by following three processes as shown in Figure 4.

First, an anisotropic amorphous layer lies adjacent to the crystallite with the interface perpendicular to the $X_{3}$ axes and the resultant system is termed as phase I. Secondly, an anisotropic amorphous layer with fraction length $1-\mu$ is attached to the structure of phase I in a plane normal to the $X_{1}$ direction to construct phase II. The final phase III can be constructed by adding an anisotropic amorphous layer with fraction length $1-\mu$ to phase II. This procedure was represented elsewhere in detail. ${ }^{15,16,23}$ For simultaneous biaxially stretched films, this model corresponds to a series model at $\mu=1$, while it corresponds to a parallel model at $\delta=1$. In following discussion, some final equations are described to shorten this paper. Complicated mathematical derivation for the composite procedure in Figure 4 was eliminated, since such treatment is similar to that of crystal lattice modulus reported elsewhere. ${ }^{7}$

In accordance with the mathematical procedure of the generalized Hook's law, Young's modulus in the stretching direction (the $X_{1}$ and $X_{2}$ directions) can be given by

$$
\begin{aligned}
E= & \frac{\mu}{S_{11}^{\mathrm{II}}}+\frac{1-\mu}{S_{11}^{\mathrm{av}}} \\
& \left.+\frac{\left\{\frac{S_{13}^{\mathrm{II}}}{S_{11}^{\mathrm{II}}}-\frac{S_{13}^{\mathrm{av}}}{S_{11}^{\mathrm{av}}}\right\}^{\mathrm{II}}\left\{\frac{S_{13}^{\mathrm{II}}}{\mu}\right\}+\frac{S_{33}^{\mathrm{av}}}{1-\mu}\left\{1-\frac{S_{13}^{\mathrm{av}}}{S_{11}^{\mathrm{II}} S_{33}^{\mathrm{av}}}\right\}}{1-S_{33}^{\mathrm{av}}}\right\}
\end{aligned}
$$

In eq $1, S_{i j}^{\mathrm{II}}$ is given as a function of $S_{i j}^{\mathrm{I}}$, $S_{i j}^{\mathrm{av}}$ and $\mu$ and $S_{i j}^{\mathrm{I}}$ is also given as a function of $S_{i j}^{\mathrm{cv}}, S_{i j}^{\mathrm{av}}$ and $\delta$ in which $S_{i j}^{\mathrm{cv}}$, and $S_{i j}^{\mathrm{av}}$ are the average compliances of crystal the amorphous phases, respectively. The complicated relationship was represented by Matsuo elsewhere. ${ }^{7}$

The relation between the intrinsic compliance of the structural unit and the bulk compliance is given as angular relationship in Figure 5. Figure 5 shows Cartesian coordinate $0-U_{1} U_{2} U_{3}$ fixed within a structural unit, with respect to another Cartesian coordinate $0-X_{1} X_{2} X_{3}$ fixed in a bulk specimen. The $U_{3}$ axis may be taken along the $c$-axis. For simultaneous biaxially stretched films, crystallites have a random orientation around the $X_{3}$ axis (the film thickness direction). The orienta-
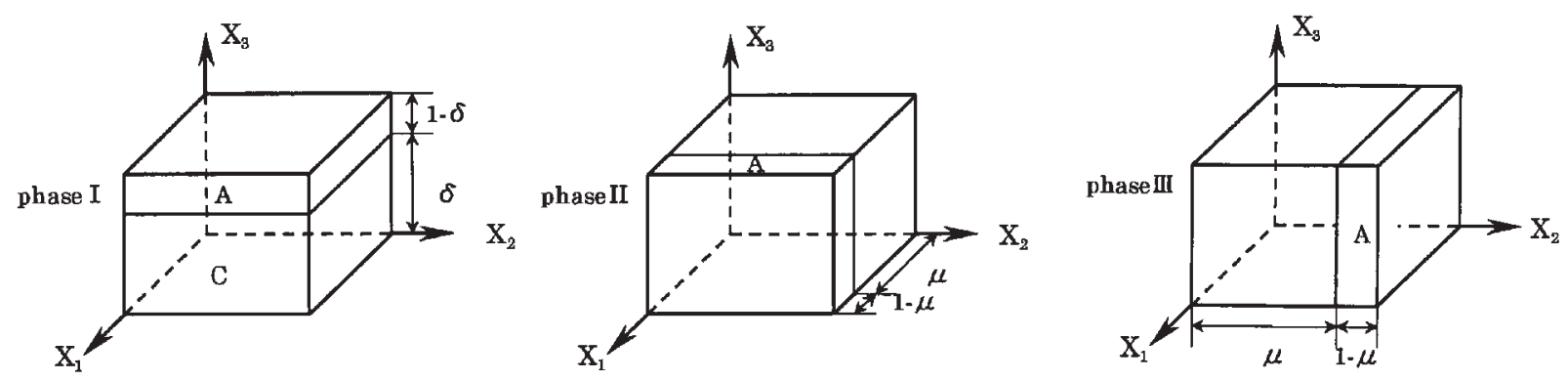

Figure 4. A procedure to construct a model in Figure 3 (1) amorphous phase attached to the $X_{3}$ face of the crystallite to construct phase I. (2) amorphous phase attached to the $X_{1}$ face of the phase I to construct phase II. (3) amorphous phase attached to the $X_{2}$ face of the phase II to construct phase III. 


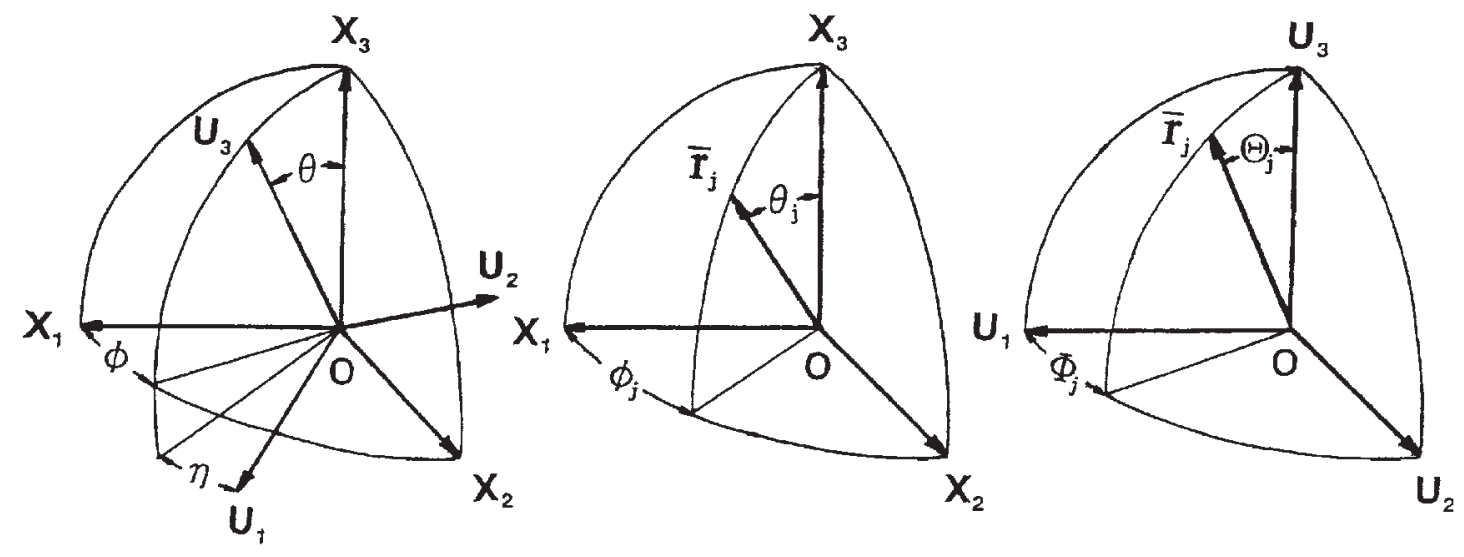

(a)

(b)

(c)

Figure 5. Cartesian coordinates illustrating the gometrical relation: (a) Euler angles $\theta$ and $\eta$ which specify the orientation of coordinate $0-U_{1} U_{2} U_{3}$ of structural unit with respect to coordinate $0-X_{1} X_{2} X_{3}$ of specimen, (b) Angles $\theta_{j}$ and $\phi_{j}$ which specify the orientation of the given $j$ th axis of the structural unit with respect to the coordinate $0-X_{1} X_{2} X_{3}$, (c) Angles $\Theta_{j}$ and $\Phi_{j}$ which specify the orientation of $j$ th axis of the structural unit with respect to the coordinate $0-U_{1} U_{2} U_{3}$.

tion of the structural unit within the space of the film specimen may be specified by using three Euler angles, $\phi, \theta$ and $\eta$. The angles $\theta$ and $\phi$, which define the orientation of $U_{3}$ axis of the unit within the space, are polar and azimuthal angles, respectively, and $\eta$ specifies the rotation of the unit around its own $U_{3}$ axis.

The tensor description between the intrinsic compliance of the structural unit and the bulk compliance is given by

$$
\begin{aligned}
& S_{i j k l}^{\mathrm{cv}}=\sum_{r=1}^{3} \sum_{q=1}^{3} \sum_{p=1}^{3} \sum_{o=1}^{3}\left\langle a_{i o} a_{j p} a_{k q} a_{l r}\right\rangle_{\mathrm{cv}} S_{o p q r}^{\mathrm{co}} \\
& S_{i j k l}^{\mathrm{av}}=\sum_{r=1}^{3} \sum_{q=1}^{3} \sum_{p=1}^{3} \sum_{o=1}^{3}\left\langle a_{i o} a_{j p} a_{k q} a_{l r}\right\rangle_{\mathrm{av}} S_{o p q r}^{\mathrm{ao}}
\end{aligned}
$$

where $S_{i j k l}^{\mathrm{cv}}$ and $S_{i j k l}^{\mathrm{av}}$ are bulk compliance of the crystal and amorphous phases, respectively, and $S_{o p q r}^{\mathrm{co}}$ and $S_{i j k l}^{\mathrm{ao}}$ are their intrinsic compliance. $a_{i o}$ is, for example, the direction cosine of the $u_{\mathrm{o}}$ axis with respect to the $X_{i}$ axis, which is given from the geometrical arrangements. Average values of the crystal phase in eq 2, $\left\langle a_{i o} a_{j p} a_{k q} a_{l r}\right\rangle_{\mathrm{cv}}$, is given by ${ }^{5-7}$

$$
\begin{aligned}
& \left\langle a_{i o} a_{j p} a_{k q} a_{l r}\right\rangle_{\mathrm{cv}} \\
& =\int_{0}^{2 \pi} \int_{0}^{2 \pi} \int_{0}^{\pi} \varpi(\theta, \eta) a_{i o} a_{j p} a_{k q} a_{l r} \sin \theta \mathrm{d} \theta \mathrm{d} \phi \mathrm{d} \eta
\end{aligned}
$$

where $\varpi(\theta, \eta)$ is the orientation distribution function of the crystal unit $0-U_{1} U_{2} U_{3}$ with respect to the coordinate $0-X_{1} X_{2} X_{3}$ in Figure 5. On the other hand, average values of the amorphous phase in eq 3 , $\left\langle a_{i o} a_{j p} a_{k q} a_{l r}\right\rangle_{\mathrm{av}}$, are given by

$$
\begin{aligned}
& \left\langle a_{i o} a_{j p} a_{k q} a_{l r}\right\rangle_{\mathrm{av}} \\
& =\int_{0}^{2 \pi} \int_{0}^{2 \pi} \int_{0}^{\pi} \varpi_{\mathrm{am}}(\theta) a_{i o} a_{j p} a_{k q} a_{l r} \sin \theta \mathrm{d} \theta \mathrm{d} \phi \mathrm{d} \eta
\end{aligned}
$$

where $\varpi_{\mathrm{am}}(\theta)$ is an orientation distribution function of the amorphous chain segments. The generalized orientation factors $F_{l 0 n}$ and $G_{l 0 n}$ of the crystal phase and $F_{l 00}^{\mathrm{am}}$ of the amorphous phases can be represented by using $\varpi(\theta, \eta)$ and $\varpi_{\mathrm{am}}(\theta)$, respectively, in the case when both the structural units have an uniaxial orientation around the $X_{3}$ axis (film thickness direction): ${ }^{1}$

$$
\begin{gathered}
F_{l 0 n}=\int_{0}^{2 \pi} \int_{0}^{2 \pi} \int_{0}^{2 \pi} \varpi(\theta, \eta) P_{l}^{n}(\cos \theta) \\
\times \cos n \eta \sin \theta \mathrm{d} \theta \mathrm{d} \phi \mathrm{d} \eta \\
G_{l 0 n}=\int_{0}^{2 \pi} \int_{0}^{2 \pi} \int_{0}^{2 \pi} \varpi(\theta, \eta) P_{l}^{n}(\cos \theta) \\
\quad \times \sin n \eta \sin \theta \mathrm{d} \theta \mathrm{d} \phi \mathrm{d} \eta
\end{gathered}
$$

and

$$
F_{l 00}^{\mathrm{am}}=\int_{0}^{2 \pi} \int_{0}^{2 \pi} \int_{0}^{2 \pi} \varpi(\theta) P_{l}(\cos \theta) \sin \theta \mathrm{d} \theta \mathrm{d} \phi \mathrm{d} \eta
$$

where $P_{l}^{n}(\cos \theta)$ and $P_{l}(\cos \theta)$ are the associated Legendre polynomial and the Legendre polynomial, respectively and $l$ must be even and $n$ takes even and odd. Incidentally, for an orthorhombic crystal form 
like PE, $F_{l 0 n}\left(n\right.$ : even) and $G_{l 0 n}=0$. For a monoclinic crystal unit like PVA and nylon $6, F_{l 0 n}(n$ : even) and $G_{l 0 n}\left(n\right.$ : even). ${ }^{14}$ The elastic compliance $S_{i j k l}$ represented as a tensor quantity may be related to $S_{u v}$ by ma- trix. ${ }^{5-7}$ Using eqs $2-8, S_{u v}^{\mathrm{cv}}$ of the crystal unit and $S_{u v}^{\mathrm{av}}$ of the amorphous unit in the stretching direction may be given as follows:

For crystal phase:

$$
\begin{aligned}
S_{11}^{\mathrm{cv}}= & S_{22}^{\mathrm{cv}}=\frac{3}{64} S_{11}^{\mathrm{co}}\left(\frac{1}{105} F_{404}-\frac{8}{105} F_{402}-\frac{32}{21} F_{202}+\frac{24}{35} F_{400}+\frac{64}{21} F_{200}+\frac{64}{15}\right) \\
& +\frac{3}{64} S_{22}^{\mathrm{co}}\left(\frac{1}{105} F_{404}+\frac{8}{105} F_{402}+\frac{32}{21} F_{202}+\frac{24}{35} F_{400}+\frac{64}{21} F_{200}+\frac{64}{15}\right)+S_{33}^{\mathrm{co}}\left(\frac{3}{35} F_{400}-\frac{2}{7} F_{200}+\frac{1}{5}\right) \\
& -\frac{1}{64}\left(2 S_{12}^{\mathrm{co}}+S_{66}^{\mathrm{co}}\right)\left(\frac{1}{35} F_{404}-\frac{24}{35} F_{400}-\frac{64}{21} F_{200}-\frac{64}{15}\right) \\
& +\frac{1}{8}\left(2 S_{13}^{\mathrm{co}}+S_{55}^{\mathrm{co}}\right)\left(\frac{1}{35} F_{402}-\frac{2}{21} F_{202}-\frac{12}{35} F_{400}-\frac{4}{21} F_{200}+\frac{8}{15}\right) \\
& -\frac{1}{8}\left(2 S_{23}^{\mathrm{co}}+S_{44}^{\mathrm{co}}\right)\left(\frac{1}{35} F_{402}-\frac{2}{21} F_{202}+\frac{12}{35} F_{400}+\frac{4}{21} F_{200}-\frac{8}{15}\right) \\
& -\frac{1}{32} S_{16}^{\mathrm{co}}\left(\frac{1}{35} G_{404}-\frac{4}{35} G_{402}-\frac{16}{7} G_{202}\right)-\frac{3}{112} S_{15}^{\mathrm{co}}\left(\frac{1}{15} F_{403}-\frac{6}{5} F_{401}-\frac{16}{3} F_{201}\right) \\
& +\frac{1}{32} S_{26}^{\mathrm{co}}\left(\frac{1}{35} G_{404}+\frac{4}{35} G_{402}+\frac{16}{7} G_{202}\right)-\frac{1}{7} S_{35}^{\mathrm{co}}\left(\frac{3}{10} F_{401}-F_{201}\right) \\
& -\frac{3}{112} S_{24}^{\mathrm{co}}\left(\frac{1}{15} G_{403}+\frac{6}{5} G_{401}+\frac{16}{3} G_{201}\right)+\frac{1}{7} S_{34}^{\mathrm{co}}\left(\frac{3}{10} G_{401}-G_{201}\right) \\
& +\frac{1}{112}\left(S_{14}^{\mathrm{co}}+S_{56}^{\mathrm{co}}\right)\left(\frac{1}{5} G_{403}-\frac{6}{5} G_{401}-\frac{16}{3} G_{201}\right) \\
& +\frac{1}{112}\left(S_{25}^{\mathrm{co}}+S_{46}^{\mathrm{co}}\right)\left(\frac{1}{5} F_{403}+\frac{6}{5} F_{401}+\frac{16}{3} F_{201}\right)-\frac{1}{14}\left(S_{36}^{\mathrm{co}}+S_{45}^{\mathrm{co}}\right)\left(\frac{1}{10} G_{402}-\frac{1}{3} G_{202}\right)
\end{aligned}
$$$$
S_{33}^{\mathrm{cv}}=\frac{1}{8} S_{11}^{\mathrm{co}}\left(\frac{1}{105} F_{404}-\frac{8}{105} F_{402}+\frac{8}{7} F_{202}+\frac{24}{35} F_{400}-\frac{16}{7} F_{200}+\frac{8}{5}\right)
$$$$
+\frac{1}{8} S_{22}^{\mathrm{co}}\left(\frac{1}{105} F_{404}+\frac{8}{105} F_{402}-\frac{8}{7} F_{202}+\frac{24}{35} F_{400}-\frac{16}{7} F_{200}+\frac{8}{5}\right)+S_{33}^{\mathrm{co}}\left(\frac{8}{35} F_{400}+\frac{4}{7} F_{200}+\frac{1}{5}\right)
$$$$
-\frac{1}{8}\left(2 S_{12}^{\mathrm{co}}+S_{66}^{\mathrm{co}}\right)\left(\frac{1}{105} F_{404}-\frac{8}{35} F_{400}+\frac{16}{21} F_{200}-\frac{8}{15}\right)
$$$$
+\frac{1}{2}\left(2 S_{13}^{\mathrm{co}}+S_{55}^{\mathrm{co}}\right)\left(\frac{2}{105} F_{402}+\frac{1}{21} F_{202}-\frac{8}{35} F_{400}+\frac{2}{21} F_{200}+\frac{2}{15}\right)
$$$$
-\frac{1}{2}\left(2 S_{23}^{\mathrm{co}}+S_{44}^{\mathrm{co}}\right)\left(\frac{2}{105} F_{402}+\frac{1}{21} F_{202}+\frac{8}{35} F_{400}-\frac{2}{21} F_{200}-\frac{2}{15}\right)
$$$$
-\frac{1}{28} S_{16}^{\mathrm{co}}\left(\frac{1}{15} G_{404}+\frac{4}{15} G_{402}+4 G_{202}\right)-\frac{1}{14} S_{15}^{\mathrm{co}}\left(\frac{1}{15} F_{403}-\frac{6}{5} F_{401}+4 F_{201}\right)
$$$$
+\frac{1}{28} S_{26}^{\mathrm{co}}\left(\frac{1}{15} G_{404}+\frac{4}{15} G_{402}-4 G_{202}\right)-\frac{2}{7} S_{35}^{\mathrm{co}}\left(\frac{2}{5} F_{401}+F_{201}\right)
$$$$
-\frac{1}{14} S_{24}^{\mathrm{co}}\left(\frac{1}{15} G_{403}+\frac{6}{5} G_{401}-4 G_{201}\right)+\frac{2}{7} S_{34}^{\mathrm{co}}\left(\frac{2}{5} G_{401}+G_{201}\right)
$$$$
+\frac{1}{14}\left(S_{14}^{\mathrm{co}}+S_{56}^{\mathrm{co}}\right)\left(\frac{1}{15} G_{403}-\frac{2}{5} G_{401}+\frac{4}{3} G_{201}\right)
$$$$
+\frac{1}{14}\left(S_{25}^{\mathrm{co}}+S_{46}^{\mathrm{co}}\right)\left(\frac{1}{15} F_{403}+\frac{2}{5} F_{401}-\frac{4}{3} F_{201}\right)-\frac{1}{21}\left(S_{36}^{\mathrm{co}}+S_{45}^{\mathrm{co}}\right)\left(\frac{2}{5} G_{402}+G_{202}\right)
$$ 


$$
\begin{aligned}
& S_{12}^{\mathrm{cv}}=\frac{1}{64} S_{11}^{\mathrm{co}}\left(\frac{1}{105} F_{404}-\frac{8}{105} F_{402}-\frac{32}{21} F_{202}+\frac{24}{35} F_{400}+\frac{64}{21} F_{200}+\frac{64}{15}\right) \\
& -\frac{1}{32} S_{12}^{\mathrm{co}}\left(\frac{1}{105} F_{404}-\frac{8}{35} F_{400}-\frac{320}{21} F_{200}-\frac{128}{15}\right) \\
& +\frac{1}{4} S_{13}^{\mathrm{co}}\left(\frac{1}{105} F_{402}-\frac{10}{21} F_{202}-\frac{4}{35} F_{400}-\frac{20}{21} F_{200}+\frac{16}{15}\right) \\
& +\frac{1}{112} S_{14}^{\mathrm{co}}\left(\frac{1}{15} G_{403}-\frac{2}{5} G_{401}-\frac{80}{3} G_{201}\right)-\frac{1}{112} S_{15}^{\mathrm{co}}\left(\frac{1}{15} F_{403}-\frac{6}{5} F_{401}-\frac{16}{3} F_{201}\right) \\
& -\frac{1}{672} S_{16}^{\mathrm{co}}\left(\frac{1}{5} G_{404}-\frac{4}{5} G_{402}-16 G_{202}\right) \\
& +\frac{1}{64} S_{22}^{\mathrm{co}}\left(\frac{1}{105} F_{404}+\frac{8}{105} F_{402}+\frac{32}{21} F_{202}+\frac{24}{35} F_{400}+\frac{64}{21} F_{200}+\frac{64}{15}\right) \\
& -\frac{1}{4} S_{23}^{\mathrm{co}}\left(\frac{1}{105} F_{402}-\frac{10}{21} F_{202}+\frac{4}{35} F_{400}+\frac{20}{21} F_{200}-\frac{16}{15}\right) \\
& -\frac{1}{112} S_{24}^{\mathrm{co}}\left(\frac{1}{15} G_{403}+\frac{6}{5} G_{401}+\frac{16}{3} G_{201}\right)+\frac{1}{112} S_{25}^{\mathrm{co}}\left(\frac{1}{15} F_{403}+\frac{2}{5} F_{401}+\frac{80}{3} F_{201}\right) \\
& +\frac{1}{672} S_{26}^{\mathrm{co}}\left(\frac{1}{5} G_{404}+\frac{4}{5} G_{402}+16 G_{202}\right)+\frac{1}{3} S_{33}^{\mathrm{co}}\left(\frac{3}{35} F_{400}-\frac{2}{7} F_{200}+\frac{1}{5}\right) \\
& +\frac{1}{14} S_{34}^{\mathrm{co}}\left(\frac{1}{5} G_{401}-\frac{2}{3} G_{201}\right)-\frac{1}{14} S_{35}^{\mathrm{co}}\left(\frac{1}{5} F_{401}-\frac{2}{3} F_{201}\right)-\frac{1}{84} S_{36}^{\mathrm{co}}\left(\frac{1}{5} G_{402}-10 G_{202}\right) \\
& -\frac{1}{8} S_{44}^{\mathrm{co}}\left(\frac{1}{105} F_{402}+\frac{4}{21} F_{202}+\frac{4}{35} F_{400}-\frac{8}{21} F_{200}+\frac{4}{15}\right) \\
& -\frac{1}{84} S_{45}^{\mathrm{co}}\left(\frac{1}{5} G_{402}+4 G_{202}\right)+\frac{1}{112} S_{46}^{\mathrm{co}}\left(\frac{1}{15} F_{403}+\frac{2}{5} F_{401}-\frac{32}{3} F_{201}\right) \\
& +\frac{1}{8} S_{55}^{\mathrm{co}}\left(\frac{1}{105} F_{402}+\frac{4}{21} F_{202}-\frac{4}{35} F_{400}+\frac{8}{21} F_{200}-\frac{4}{15}\right) \\
& +\frac{1}{112} S_{56}^{\mathrm{co}}\left(\frac{1}{15} G_{403}-\frac{2}{5} G_{401}+\frac{32}{3} G_{201}\right)-\frac{1}{64} S_{66}^{\mathrm{co}}\left(\frac{1}{105} F_{404}-\frac{8}{35} F_{400}+\frac{128}{21} F_{200}+\frac{32}{15}\right) \\
& S_{13}^{\mathrm{cv}}=S_{23}^{\mathrm{cv}}=-\frac{1}{16} S_{11}^{\mathrm{co}}\left(\frac{1}{105} F_{404}-\frac{8}{105} F_{402}-\frac{4}{21} F_{202}+\frac{24}{35} F_{400}+\frac{8}{21} F_{200}-\frac{16}{15}\right) \\
& +\frac{1}{8} S_{12}^{\mathrm{co}}\left(\frac{1}{105} F_{404}-\frac{8}{35} F_{400}-\frac{40}{21} F_{200}+\frac{32}{15}\right) \\
& -\frac{1}{4} S_{13}^{\mathrm{co}}\left(\frac{4}{105} F_{402}-\frac{5}{21} F_{202}-\frac{16}{35} F_{400}-\frac{10}{21} F_{200}+\frac{16}{15}\right) \\
& -\frac{1}{28} S_{14}^{\mathrm{co}}\left(\frac{1}{15} G_{403}-\frac{2}{5} G_{401}-\frac{10}{3} G_{201}\right)+\frac{1}{28} S_{15}^{\mathrm{co}}\left(\frac{1}{15} F_{403}-\frac{6}{5} F_{401}-\frac{2}{3} F_{201}\right) \\
& +\frac{1}{168} S_{16}^{\mathrm{co}}\left(\frac{1}{5} G_{404}-\frac{4}{5} G_{402}-2 G_{202}\right) \\
& -\frac{1}{16} S_{22}^{\mathrm{co}}\left(\frac{1}{105} F_{404}+\frac{8}{105} F_{402}+\frac{4}{21} F_{202}+\frac{24}{35} F_{400}+\frac{8}{21} F_{200}-\frac{16}{15}\right) \\
& +\frac{1}{4} S_{23}^{\mathrm{co}}\left(\frac{4}{105} F_{402}-\frac{5}{21} F_{202}+\frac{16}{35} F_{400}+\frac{10}{21} F_{200}+\frac{16}{15}\right) \\
& +\frac{1}{28} S_{24}^{\mathrm{co}}\left(\frac{1}{15} G_{403}+\frac{6}{5} G_{401}+\frac{2}{3} G_{201}\right)-\frac{1}{28} S_{25}^{\mathrm{co}}\left(\frac{1}{15} F_{403}+\frac{2}{5} F_{401}+\frac{10}{3} F_{201}\right) \\
& +\frac{1}{168} S_{26}^{\mathrm{co}}\left(\frac{1}{5} G_{404}+\frac{4}{5} G_{402}+2 G_{202}\right)-S_{33}^{\mathrm{co}}\left(\frac{4}{35} F_{400}-\frac{1}{21} F_{200}-\frac{1}{15}\right)
\end{aligned}
$$




$$
\begin{aligned}
& -\frac{1}{14} S_{34}^{\mathrm{co}}\left(\frac{4}{5} G_{401}-\frac{1}{3} G_{201}\right)+\frac{1}{14} S_{35}^{\mathrm{co}}\left(\frac{4}{5} F_{401}-\frac{1}{3} F_{201}\right)+\frac{1}{42} S_{36}^{\mathrm{co}}\left(\frac{2}{5} G_{402}-\frac{5}{2} G_{202}\right) \\
& +\frac{1}{4} S_{44}^{\mathrm{co}}\left(\frac{2}{105} F_{402}+\frac{1}{21} F_{202}+\frac{8}{35} F_{400}-\frac{2}{21} F_{200}-\frac{2}{15}\right) \\
& +\frac{1}{42} S_{45}^{\mathrm{co}}\left(\frac{2}{5} G_{402}+G_{202}\right)-\frac{1}{28} S_{46}^{\mathrm{co}}\left(\frac{1}{15} F_{403}+\frac{2}{5} F_{401}-\frac{4}{3} F_{201}\right) \\
& -\frac{1}{4} S_{55}^{\mathrm{co}}\left(\frac{2}{105} F_{402}+\frac{1}{21} F_{202}-\frac{8}{35} F_{400}+\frac{2}{21} F_{200}+\frac{2}{15}\right) \\
& -\frac{1}{28} S_{56}^{\mathrm{co}}\left(\frac{1}{15} G_{403}-\frac{2}{5} G_{401}+\frac{4}{3} G_{201}\right)+\frac{1}{16} S_{66}^{\mathrm{co}}\left(\frac{1}{105} F_{404}-\frac{8}{35} F_{400}+\frac{16}{21} F_{200}-\frac{8}{15}\right)
\end{aligned}
$$

For amorphous phase:

$$
\begin{aligned}
S_{11}^{\mathrm{av}}= & S_{22}^{\mathrm{av}}=\frac{3}{64} S_{11}^{\mathrm{ao}}\left(\frac{24}{35} F_{400}^{\mathrm{am}}+\frac{64}{21} F_{200}^{\mathrm{am}}+\frac{64}{15}\right) \\
& +\frac{3}{64} S_{22}^{\mathrm{ao}}\left(\frac{24}{35} F_{400}^{\mathrm{am}}+\frac{64}{21} F_{200}^{\mathrm{am}}+\frac{64}{15}\right) \\
& +S_{33}^{\mathrm{ao}}\left(\frac{3}{35} F_{400}^{\mathrm{am}}-\frac{2}{7} F_{200}^{\mathrm{am}}+\frac{1}{5}\right) \\
& +\frac{1}{64}\left(2 S_{12}^{\mathrm{ao}}+S_{66}^{\mathrm{ao}}\right)\left(\frac{24}{35} F_{400}^{\mathrm{am}}+\frac{64}{21} F_{200}^{\mathrm{am}}+\frac{64}{15}\right) \\
& +\frac{1}{8}\left(2 S_{13}^{\mathrm{ao}}+S_{55}^{\mathrm{ao}}\right)\left(-\frac{12}{35} F_{400}^{\mathrm{am}}-\frac{4}{21} F_{200}^{\mathrm{am}}+\frac{8}{15}\right) \\
& -\frac{1}{8}\left(2 S_{23}^{\mathrm{ao}}+S_{44}^{\mathrm{ao}}\right)\left(\frac{12}{35} F_{400}^{\mathrm{am}}+\frac{4}{21} F_{200}^{\mathrm{am}}-\frac{8}{15}\right)
\end{aligned}
$$

The other compliances $S_{u v}^{\text {ao }}$ can be obtained by using $F_{404}=F_{403}=F_{402}=F_{401}=0, \quad F_{202}=F_{201}=0$, $G_{404}=G_{403}=G_{402}=G_{401}=0, \quad G_{202}=G_{201}=0$ and by replacing $F_{200}$ and $F_{400}$ into $F_{200}^{\text {am }}$ and $F_{400}^{\text {am }}$, respectively as well as by replacing $S_{i j}^{\mathrm{co}}$ into $S_{i j}^{\mathrm{ao}}$.

Here the orientation factors in eqs $9-13$ may be given by

$$
\begin{aligned}
& F_{l 0 n}=\left\langle P_{l}^{n}(\cos \theta) \cos n \eta\right\rangle \\
& F_{l 00}^{\mathrm{am}}=\left\langle P_{l 00}(\cos \theta)\right\rangle \\
& G_{l 0 n}=\left\langle P_{l}^{n}(\cos \theta) \sin n \eta\right\rangle
\end{aligned}
$$

The generalized fourth and second order orientation factors obtained for the PET film with $4 \times 4$ are listed in Table III. These values are estimated on the basis of the orientation distribution function of crystallites in the previous paper. ${ }^{15}$ The representation of eqs 913 for PET crystal unit is much complicated in comparison with for PE with an orthorhombic crystal unit and for polypropylene (PP), PVA and nylon 6 with a monoclinic crystal unit. The problem that now arises has been how the value of the intrinsic compliances $S_{u v}^{\mathrm{co}}$ and $S_{u v}^{\mathrm{ao}}$ of PET can be determined theoretically. The values as the pioneering work were obtained by Tashiro et al. ${ }^{25}$ using $\mathbf{B}$ matrix at absolute temperature. According to their paper,

$$
S_{u v}^{\mathrm{co}}=\left|\begin{array}{rrrrrr}
2.986 & -2.033 & -0.2279 & 3.106 & -5.700 & -5.019 \\
& 2.317 & 0.1953 & -2.255 & 6.513 & -4.136 \\
& & 0.1130 & -0.4718 & 1.220 & -0.4098 \\
& & & 9.250 & -9.768 & 6.562 \\
& & & & 5.023 & -6.690 \\
& & & & & 1.868
\end{array}\right| \times 10^{-1} / \mathrm{GPa}
$$

Because of the lack of inharmonic effects, the crystal lattice modulus, $88.5 \mathrm{GPa}$, along the chain direction given by $1 / S_{33}^{\mathrm{co}}$ is much smaller than the crystal lattice modulus along the direction, $c a$. $118 \mathrm{GPa}$, calculated by using the somewhat complicated treatment,$^{19}$ on the basis of the crystal lattice modulus of the (105) plane at room temperature (see Table II).

The intrinsic elastic compliances $S_{u v}^{\mathrm{ao}}$ of the amorphous phase needed in the numerical calculation is not quite certain. In accordance with the previous method, ${ }^{3-7}$ however, the values can be estimated as a crude approximation, assuming that the following relation between the potential energy $P(r)$ of Lennard-Jones and the atomic or molecular distance $r$ holds for a noncrystalline chain

$$
P(r)=-\frac{c}{r^{n}}+\frac{d}{r^{m}}
$$


Table III. The generalized forth and second order orientation factors estimatied for the PET film stretched up to $4 \times 4^{15}$

\begin{tabular}{lccccccc}
\hline$F_{400}$ & 0.2998 & & & $F_{200}$ & -0.493 & $G_{201}$ & -0.0812 \\
$F_{401}$ & 0.1121 & $G_{401}$ & -0.5467 & $F_{201}$ & 0.1022 & $G_{202}$ & -0.5201 \\
$F_{402}$ & -2.421 & $G_{402}$ & 1.721 & $F_{202}$ & 1.436 & & \\
$F_{403}$ & 4.485 & $G_{403}$ & 3.397 & & & & \\
$F_{404}$ & 27.30 & $G_{404}$ & -24.38 & & & & \\
\hline
\end{tabular}

where $m=9-12$ and $n=1$ or 6 for an ionic or molecular crystal, respectively. Then, neglecting the repulsion term in eq 18 for $r$ greater than the equilibrium distance, the elastic compliance $S_{11}^{\mathrm{ao}}$ and $S_{22}^{\mathrm{ao}}$ may be estimated by taking the second-order derivative of $P(r)$. Then, ${ }^{5}$

$$
S_{11}^{\mathrm{ao}}=S_{22}^{\mathrm{ao}}=\left\{\frac{\rho_{\mathrm{c}}}{\rho_{\mathrm{a}}}\right\}^{4} S_{11}^{c^{\prime}}
$$

where $S_{11}^{c^{\prime}}$ corresponds to $S_{11}^{\mathrm{cv}}$ at $\theta=0^{\circ}$ in Figure 5, $\rho_{\mathrm{c}}$ and $\rho_{\mathrm{a}}$ are the intrinsic densities of the crystalline and amorphous phases, respectively. $\rho_{\mathrm{c}}$ and $\rho_{\mathrm{a}}$ are given as 1.455 and $1.335 \mathrm{~g} / \mathrm{cm}^{3}$, respectively. ${ }^{26}$ On the other hand, the expansion of the amorphous phase is assumed to occur only along the lateral direction of the polymer chain, not lengthwise. The compliance $S_{33}^{\text {ao }}$ may be estimated by assuming that the modulus along the chain axis is proportional to the number of chain molecules in the unit area perpendicular to the chain direction, and that the molecules is independent of temperature. Thus we have

$$
S_{33}^{\mathrm{ao}}=\left\{\frac{\rho_{\mathrm{c}}}{\rho_{\mathrm{a}}}\right\} S_{33}^{\mathrm{co}}
$$

The other compliances are given by Hibi et al. ${ }^{17,18}$ That is,

$$
\begin{aligned}
& S_{44}^{\mathrm{ao}}=S_{55}^{\mathrm{ao}} \\
& S_{12}^{\mathrm{ao}}=-v_{12}^{\mathrm{a} o} S_{11}^{\mathrm{ao}} \\
& S_{23}^{\mathrm{ao}}=S_{13}^{\mathrm{ao}}=-v_{13}^{\mathrm{ao}} S_{33}^{\mathrm{ao}} \\
& 2 S_{11}^{\mathrm{ao}}=\left(2 S_{13}^{\mathrm{ao}}+S_{55}^{\mathrm{ao}}\right) \Omega \\
& 2 S_{11}^{\mathrm{ao}}=2 S_{12}^{\mathrm{ao}}+S_{66}^{\mathrm{ao}}
\end{aligned}
$$

The parameter $\Omega$ is first introduced by Hibi et $a l .{ }^{17,18}$ to determine the value of $S_{55}^{\text {ao }}$ as an unknown parameter. The Poisson's ratio $v_{12}^{\mathrm{ao}}\left(=v_{13}^{\mathrm{ao}}\right)$ is an unknown parameter and is set to be 0.49 or 0.33 in this paper. The former and the latter values of $v_{12}^{\text {ao }}$ are selected in the case where the mechanical property of the amorphous phase is similar to ideal rubber elasticity or somewhat tougher or glass state, respectively.

The second order orientation factor $F_{200}^{\text {am }}$ could be estimated from birefringence measurement. ${ }^{27}$ Assuming that the birefringence, $\Delta_{\mathrm{am}}$, from the amorphous phase may be estimated by subtracting the crystalline birefringence $\Delta_{\text {cry }}$ from the total birefringence $\Delta_{\text {total }}$, we have $\mathrm{e}^{28}$

$$
\Delta_{\mathrm{am}}=\frac{\Delta_{\text {total }}-X_{\mathrm{c}} \Delta_{\mathrm{c}}}{1-X_{\mathrm{c}}}
$$

where

$$
\Delta_{\mathrm{am}}=\left\{n_{33}^{\mathrm{am}}-\frac{n_{11}^{\mathrm{am}}+n_{22}^{\mathrm{am}}}{2}\right\} F_{200}^{\mathrm{am}}
$$

and

$$
\Delta_{\mathrm{c}}=\left\{n_{33}^{\mathrm{co}}-\frac{n_{11}^{\mathrm{co}}+n_{22}^{\mathrm{co}}}{2}\right\} F_{200}+\frac{n_{11}^{\mathrm{co}}-n_{22}^{\mathrm{co}}}{4} F_{202}
$$

where $X_{\mathrm{c}}$ is the degree of volume crystallinity. The parameters $n_{33}^{\text {co }}$ and $n_{11}^{\text {co }}$ are the refractive indexes along the $c$-axis and $a^{*}$-axis corresponding to the direction normal to the benzene ring. $n_{22}^{\text {co }}$ is the refractive index in the direction perpendicular to the $c$ and $a^{*}$ axes. $n_{33}^{\text {am }}$ is the refractive index normal to the benzene ring. $n_{11}^{\text {am }}$ and $n_{22}^{a m}$ are the refractive perpendicular to the above axis. All the values were calculated by Sakaguchi et $a l .{ }^{29}$ in the usual way by assuming the atomic arrangements within the crystal unit and the values of the bond polarizabilities according to Bunn and Daubeny ${ }^{30}$ and by neglecting the uncertain effect of the internal field within the crystal and of secondary bonds upon the principal polarizabilities. They are given by ${ }^{28}$

$$
\begin{aligned}
& n_{33}^{\mathrm{co}}=1.806 \\
& n_{22}^{\mathrm{co}}=1.733 \\
& n_{11}^{\mathrm{co}}=1.398 \\
& n_{33}^{\mathrm{am}}=1.362 \\
& n_{11}^{\mathrm{am}}=n_{22}^{\mathrm{am}}=1.687
\end{aligned}
$$

Since $n_{33}^{\mathrm{am}}<n_{22}^{\mathrm{am}}, F_{200}^{\mathrm{am} \prime}$ corresponds to the second order orientation factor of the direction normal to the benzene ring with respect to the stretching direction. In the case when the amorphous chain axes orient randomly around their own axes, the orientation factors of the other two axes become equivalent with respect to the stretching direction. Then, the orientation factor of the amorphous chain axes may be given as follows: ${ }^{28}$

$$
F_{200}^{\mathrm{am}}=-2 F_{200}^{\mathrm{am} \prime}
$$

In the present specimen, the value of $F_{200}^{\mathrm{am}}$ was -0.4998 , indicating that the amorphous chains are almost oriented parallel to the film surface.

To calculate the mechanical properties according to eqs 1-26, the fourth-order orientation factor $F_{400}^{\mathrm{am}}$ of the amorphous chain segments must be estimated. Unfortunately, this factor cannot be obtained from birefringence measurements and therefore must be calculated by assuming a common function as an orientation function of amorphous chain segments. In this paper, the mean fourth power of direction $\left\langle\cos ^{4} \theta\right\rangle_{\text {am }}$ was calculated from an inversely super- 


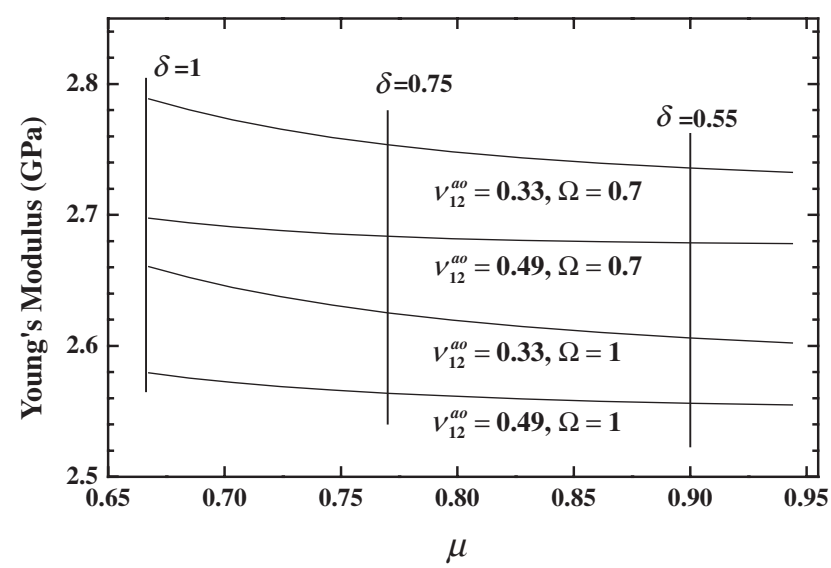

Figure 6. $\mu$-Dependence of Young's modulus $E$ calculated at $v_{12}^{\mathrm{ao}}\left(=v_{13}^{\mathrm{ao}}\right)=0.33$ and 0.49 in the case of $\Omega=0.7$ and 1 .

posed Gaussian function given elsewhere ${ }^{31}$ and the value of $F_{400}^{\mathrm{am}}$ was 0.375 .

Based on the procedure discussed above, the values of average elastic compliance $S_{i j}^{\mathrm{cv}}$ and $S_{i j}^{\mathrm{av}}$ of the crystal and amorphous phases can be determined. By using these values, $S_{i j}^{\mathrm{I}}$ and $S_{i j}^{\mathrm{II}}$ are calculated on the procedure described elsewhere ${ }^{7}$ and consequently the Young's modulus can be calculated by using eq 1 .

Figure 6 shows the $\mu$-dependence of Young's modulus $E$ calculated at $v_{12}^{\mathrm{ao}}\left(=v_{13}^{\mathrm{ao}}\right)=0.33$ and 0.49 in the case of $\Omega=0.7$ and 1 . Here $\delta$ is determined automatically by $X_{\mathrm{c}}=\mu^{2} \delta\left(X_{\mathrm{c}}\right.$ is volume crystallinity). The numerical calculation was carried out using the intrinsic crystal elastic compliance $S_{u v}^{\mathrm{co}}$ proposed by Tashiro et al. ${ }^{25}$ The value of $E$ is more sensitive to $\Omega$ than $v_{12}^{\text {ao }}$. Judging from the physical meaning of $\Omega$ in eq 21 to determine $S_{55}^{\mathrm{aa}}$, this tendency indicates the significant effect on shear modulus on Young's modulus for biaxial stretching films. Anyway, these calculated values of $E$ decreased slightly with increasing $\mu$. This means that a series model at $\delta=1$ gives higher Young's modulus, when crystallinity and molecular orientation within a bulk and the value of $\Omega$ are the same. Even so, the calculated Young's modulus is in the range from 2.55-2.79 GPa and the values are in good agreement with the experimental value at room temperature. This is probably due to the fact that the mechanical property of simultaneous biaxially stretched PET film can be represented roughly by the theory for infinitesimal deformation of an aniisotropic elastic body using 36 independent elastic compliances based on the homogeneous stress hypothesis. As another analysis, we must emphasize that the intrinsic densi$\operatorname{ty}^{26}$ of amorphous phase $\rho_{\mathrm{a}}$ is almost equal to the density of amorphous PET film at room temperature, apart from another crystalline polymers. The amorphous density of most of crystalline polymers has been obliged to adopt the value at their melt state be- cause of rapid crystallization. Accordingly, the values of real stiffness of the PET film are thought to be in good agreement with $S_{11}^{\text {ao }}$ and $S_{22}^{\text {ao }}$ calculated by eq 19 and $S_{33}^{\text {ao }}$ by eq 20, although they could not be obtained experimentally. The increase in $E^{\prime}$ with decreasing temperature, as shown in Figure 1, is postulated to be an increase in $\rho_{\mathrm{a}}$ with decreasing temperature. In other wards, the values of $\rho_{\mathrm{a}}$ and $X_{\mathrm{c}}$ decrease drastically with increasing temperature and consequently the Young's modulus $E$ and/or the storage modulus $E^{\prime}$ decrease with increasing temperature.

As listed in Table II, the crystal lattice modulus depends on temperature except along the chain direction. Accordingly, it may be postulated that the moduli of the crystal and amorphous phases decrease with increasing temperature and consequently the Young's modulus of the present film decreases. The drastic decrease in the amorphous modulus deduces from the decrease in the Young's modulus in bulk along the stretching direction as listed in Table II, since the corresponding crystal lattice modulus is independent of temperature.

Finally, we shall refer to the ultimate goal of Young's modulus of an ideal simultaneous biaxially stretched film such that the crystallinity is $100 \%$ and the $c$-axis orient to the film surface perfectly. Furthermore, the benzene rings must be oriented perfectly parallel to the film surface. Such mathematical treatment, however, is very complicated because of the difficulty in obtaining the accurate geometrical arrangement of benzene ring within the unit cell. In this paper, the benzene rings are assumed to be parallel to the $a-c$ crystal plane and then the generalized orientation factors can be obtained by substituting $\theta=90^{\circ}$ and $\eta=0^{\circ}$ into eqs 14 and 16 . Thus, we obtain $F_{200}=-1 / 2, F_{202}=3, F_{400}=3 / 8, F_{402}=-15 / 2$, $F_{404}=105$ and the other factors become zero. By the calculation at $X_{\mathrm{c}}\left(=\delta \mu^{2}\right)=1$, the ultimate value of Young's modulus for the biaxially stretching film is found to be $4.73 \mathrm{GPa}$. This value is much lower than the crystal lattice modulus along the chain direction estimated by X-ray diffraction (see Table II) and the theoretical modulus by Tashiro. ${ }^{25}$ This tendency is due to the fact that Young's modulus of a biaxially stretched film is isotropic around the film normal direction and consequently e compliance relating to sher modulus. In contrast, Young's modulus of a uniaxial film with a perfect molecular orientation depends on the compliance $S_{33}^{\mathrm{co}}$ in the chain direction.

The experimental value at $20^{\circ} \mathrm{C}$ is less than $4.73 \mathrm{GPa}$ as the ultimate value for PET, when the crystallites are oriented randomly around the film thickness. Furthermore, the Young's modulus at $-100^{\circ} \mathrm{C}$ is close to the ultimate value. This phenomenon is attributed to the theoretical value, $88.5 \mathrm{Gpa}$, 
by Tashiro $^{24}$ smaller than the crystal lattice modulus $(118 \mathrm{GPa})$ estimated from the value of (105) plane measured by X-ray diffraction (see Table II). Anyway, the predicated ultimate Young's modulus indicates the impossibility to produce high-modulus and high-strength PET sheets.

\section{CONCLUSIONS}

Young's modulus of poly(ethylene terephthalate) (PET) film was estimated by using the generalized orientation factors of crystallites and amorphous chain segments calculated from the orientation functions of crystallites and amorphous chain segments. The relationship between the elastic compliance of oriented crystallites and the generalized orientation factors for PET with a triclinic crystal unit were much complicated than that for polyethylene with an orthorhombic crystal unit and that for poly(vinyl alcohol) with a monoclinic crystal unit. The analysis of the Young's modulus was carried out on the basis of a two-phase model assuring the homogeneous stress hypothesis for a polycrystalline material. In doing so, a three-dimensional model was proposed, in which the anisotropic crystal phase is surrounded by the anisotropic amorphous phase. This model system satisfies the morphological characteristics of a polymeric system with a low degree of molecular orientation and low crystallinity such as a simultaneous biaxial stretching film. The theoretical values of elastic compliance of crystal unit of PET estimated by Tashiro were adopted to pursue theoretical calculation. The calculated Young's modulus depends on the composite mode but the difference was not considerable. The Young's modulus was in $2.55-2.79 \mathrm{GPa}$ and the values are in good agreement with the experimental results. The ultimate value of Young's modulus was estimated by assuming an ideal simultaneous biaxial stretching film with $100 \%$ crystallinity and the perfect orientation of the $c$-axis as well as that of benzene rings parallel to the film surface. Even in such an ideal case, the predicted value was less than $5 \mathrm{GPa}$. Accordingly, it was demonstrated to be impossible to produce high modulus and high strength PET sheets in terms of theoretical aspects, in spite of industrial production of high modulus and high strength polyethylene fibers and tapes.

Acknowledgment. The authors are indebted to Prof. Tashiro, Osaka University, Osaka, Japan, who has originated to pursue theoretical calculations concerning the elastic compliance of a crystal unit cell by using B-matrix. He offered the values of a PET crystal unit before his publication to help them. They express great thanks for his kindness.

\section{REFERENCES}

1. R. J. Roe and W. R. Krigbaum, J. Chem. Phys., 40, 2608 (1964).

2. R. J. Roe, J. Appl. Phys., 36, 2024 (1965).

3. S. Nomura, S. Kawabata, H. Kawai, Y. Yamaguchi, A. Fukushima, and H. Takahara, J. Polym. Sci., Part A-2: Polym. Phys., 7, 1969 (1969).

4. M. Maeda, S. Hibi, F. Ito, S. Nomura, T. Kawaguchi, and H. Kawai, J. Polym. Sci., Part A-2: Polym. Phys., 8, 1303 (1970).

5. C. Sawatari and M. Matsuo, Macromolecules, 19, 2726 (1986).

6. M. Matsuo and C. Sawatari, Macromolecules, 21, 1658 (1988).

7. M. Matsuo, Macromolecules, 23, 3261 (1990).

8. P. R. Pinnock, I. M. Ward, and J. M. Wolfe, Proc. R. Soc. London, Ser. A, 291, 267 (1966).

9. V. B. Gupta and I. M. Ward, J. Macromol. Sci., Part B: Phys., 1, 373 (1967).

10. M. Matsuo, Y. Harashina, and T. Ogita, Polym. J., 25, 319 (1993).

11. M. Matsuo, R. Sato, N. Yanagida, and Y. Shimizu, Polymer, 33, 1640 (1992).

12. M. Matsuo, R. Sato, and Y. Shimizu, Colloid Polym. Sci., 271, 11 (1993).

13. M. Matsuo, C. Sawatari, Y. Iwai, and F. Ozaki, Macromolecules, 23, 3266 (1990).

14. M. Matsuo, R. Adachi, X. Jiang, and Y. Bin, Macromolecules, 37, 1324 (2004).

15. Y. Bin, K. Oishi, K. Yoshida, T. Nakashima, and M. Matsuo, Polym. J., 36, 394 (2004).

16. W. Kuhn and F. Grun, Kolloid-Z., 101, 248 (1942).

17. S. Hibi, M. Maeda, S. Makino, S. Nomura, and S. Kawai, Sen-i Gakkaishi, 29, 79 (1973).

18. S. Hibi, M. Maeda, M. Mizuno, S. Nomura, and S. Kawai, Sen-i Gakkaishi, 29, 137 (1973).

19. M. Matsuo and C. Sawatari, Polym. J., 22, 518 (1990).

20. K. H. Illers and H. J. Breuer, Colloid Sci., 18, 1 (1963).

21. I. M. Ward, Trans. Faraday Soc., 56, 648 (1960).

22. N. Yoshihara, A. Fukushima, Y. Watanabe, A. Nakai, S. Nomura, and H. Kawai, Sen-i Gakkaishi, 37, 387 (1981).

23. M. Matsuo and C. Sawatari, Macromolecules, 21, 1653 (1988).

24. C. Sawatari and M. Matsuo, Macromolecules, 22, 2968 (1989).

25. K. Tashiro, unpublished data.

26. J. Brandrup, E. H. Immergut, and E. A. Grulke, "Polymer Handbook, 4th ed.," John Wiley \& Sons, Inc., New York, N.Y., 1999.

27. R. S. Stein and F. H. Norris, J. Polym. Sci., 21, 381 (1956).

28. M. Matsuo, M. Tamada, T. Terada, C. Sawatari, and M. Niwa, Macromolecules, 15, 988 (1982).

29. N. Sakaguchi, T. Oda, A. Nakai, and H. Kawai, Sen-i Gakkaishi, 33, 499 (1977).

30. R. de Daubeny, C. W. Q. Bunn, and C. Brown, Proc. R. Soc. London, Ser. A, 226, 531 (1954).

31. Y. Bin, Y. Tanabe, C. Nakabayashi, H. Kurose, and M. Matsuo, Polymer, 42, 1183 (2001). 